\title{
Discrimination performance in aging is vulnerable to interference and dissociable from spatial memory
}

\author{
Sarah A. Johnson, ${ }^{1}$ Patricia K. Sacks, ${ }^{1}$ Sean M. Turner, ${ }^{1}$ Leslie S. Gaynor, ${ }^{2}$ \\ Brandi K. Ormerod, ${ }^{3}$ Andrew P. Maurer, ${ }^{1,3}$ Jennifer L. Bizon, ${ }^{1}$ and Sara N. Burke ${ }^{1,4}$ \\ ${ }^{1}$ Department of Neuroscience, Evelyn F. and William L. McKnight Brain Institute; ${ }^{2}$ Department of Clinical and Health Psychology; \\ ${ }^{3}$ Department of Biomedical Engineering, University of Florida, Gainesville, Florida 32610, USA; ${ }^{4}$ Institute on Aging, University \\ of Florida, Gainesville, Florida 32610, USA
}

\begin{abstract}
Hippocampal-dependent episodic memory and stimulus discrimination abilities are both compromised in the elderly. The reduced capacity to discriminate between similar stimuli likely contributes to multiple aspects of age-related cognitive impairment; however, the association of these behaviors within individuals has never been examined in an animal model. In the present study, young and aged F344 $\times$ BN F1 hybrid rats were cross-characterized on the Morris water maze test of spatial memory and a dentate gyrus-dependent match-to-position test of spatial discrimination ability. Aged rats showed overall impairments relative to young in spatial learning and memory on the water maze task. Although young and aged learned to apply a match-to-position response strategy in performing easy spatial discriminations within a similar number of trials, a majority of aged rats were impaired relative to young in performing difficult spatial discriminations on subsequent tests. Moreover, all aged rats were susceptible to cumulative interference during spatial discrimination tests, such that error rate increased on later trials of test sessions. These data suggest that when faced with difficult discriminations, the aged rats were less able to distinguish current goal locations from those of previous trials. Increasing acetylcholine levels with donepezil did not improve aged rats' abilities to accurately perform difficult spatial discriminations or reduce their susceptibility to interference. Interestingly, better spatial memory abilities were not significantly associated with higher performance on difficult spatial discriminations. This observation, along with the finding that aged rats made more errors under conditions in which interference was high, suggests that match-to-position spatial discrimination performance may rely on extra-hippocampal structures such as the prefrontal cortex, in addition to the dentate gyrus.
\end{abstract}

[Supplemental material is available for this article.]

The majority of elderly individuals will experience memory loss that impacts daily functioning. Specifically, older adults recall less detailed information about events due to source memory deficits (McIntyre and Craik 1987; Schacter et al. 1994; Cansino 2009), experience decreased sensitivity to novelty (Fandakova et al. 2014), and show increased susceptibility to proactive interference (Hartman 1995; Pettigrew and Martin 2014; Wais and Gazzaley 2014). Parallel observations have been made in animal models of aging. Aged rats are impaired in spatial memory (Gallagher et al. 1993; Bizon et al. 2009; McQuail and Nicolle 2015), in discriminating between novel and familiar stimuli (Burke et al. 2010, 2011), and in place learning when the learned target location is in close proximity to a foil location (Gracian et al. 2013).

Age-related deficits in retrieving detailed information from episodic memory could reflect an inability to discriminate between similar stimuli, as well as repeated similar experiences. In support of this interpretation, discrimination abilities are impaired in healthy aged humans (Toner et al. 2009; Stark et al. 2010, 2013; Holden et al. 2012, 2013; Ryan et al. 2012; Reagh et al. 2014, 2016), monkeys (Burke et al. 2011), and rats (Burke et al. 2010, 2011). An intriguing aspect of these deficits is that older adults who do not show episodic memory loss can be impaired relative to young adults at difficult discriminations between similar stimuli, but not easy discriminations between

\section{Corresponding author: burkes@ufl.edu}

Article is online at http://www.learnmem.org/cgi/doi/10.1101//m.042069.116. unique stimuli (Stark et al. 2013; Reagh et al. 2014, 2016). This observation suggests that age-related discrimination deficits could precede broader episodic memory decline.

Spatial memory deficits have been considered to parallel episodic memory or recollection deficits in humans (Robitsek et al. 2008; Tomás Pereira and Burwell 2015). Therefore, a test of whether changes in discrimination ability contribute to broader changes in mnemonic ability across the lifespan would be to assess both spatial memory and spatial discrimination performance in the same cohort of aged rats. The present study examined young and aged rats' abilities to discriminate between target and foil locations on a hole-board maze using a spatial match-to-position task (Gilbert et al. 1998, 2001; Kesner and Gilbert 2006). During tests, the difficulty of each spatial discrimination trial was manipulated by decreasing the physical distance between target and foil locations. To determine whether spatial discrimination performance is associated with hippocampal-dependent spatial memory, rats were cross-characterized on the Morris water maze task (Gallagher et al. 1993; Bizon et al. 2009). To examine the extent to which declines in cholinergic transmission could explain performance differences between age groups, rats were injected

(C) 2016 Johnson et al. This article is distributed exclusively by Cold Spring Harbor Laboratory Press for the first 12 months after the full-issue publication date (see http://learnmem.cshlp.org/site/misc/terms.xhtml). After 12 months, it is available under a Creative Commons License (Attribution-NonCommercial 4.0 International), as described at http://creativecommons.org/licenses/by-nc/4.0/. 
with the acetylcholinesterase inhibitor donepezil and retested on the discrimination task.

\section{Results}

\section{Spatial memory is impaired in aged rats}

To assess age-related changes in spatial learning and memory in our experimental cohort, rats were first tested in the Morris water maze task using the standard procedures of Gallagher et al. (1993), which have been implemented in multiple rat strains used to study cognitive aging (Bizon et al. 2009; McQuail and Nicolle 2015). Young and aged rats were trained to locate a hidden platform over $8 \mathrm{~d}$, with blocks of training trials interpolated by probe trials taking place every $2 \mathrm{~d}$. Acquisition of an effective spatial search strategy was assessed based on cumulative integrated path lengths (CIPL) across successive blocks of training trials (Barnes et al. 1996; Rosenzweig et al. 1997; Burke et al. 2010). CIPL is equivalent to the cumulative search error originally reported by Gallagher et al. (1993), and reflects distance traveled in locating the platform, corrected for trial-to-trial differences in optimal path and swim speed (see Materials and Methods). A repeated-measures ANOVA comparing performance across training blocks $1-4$ revealed a significant main effect of block (Fig. $\left.1 \mathrm{~A} ; F_{(3,51)}=10.9, P<0.001\right)$. However, overall performance and change in performance across blocks did not differ based on age $(P s>0.13)$. Within subjects simple contrasts confirmed that CIPL values for block 1 were greater than those for all subsequent blocks ( $F \mathrm{~s}>8.53, P \mathrm{~s}<0.01)$. Block 1 is distinct from subsequent training blocks, as animals are at this point acquiring procedural aspects of the task and show high levels of thigmotaxis. Thus, to further examine whether aging influenced the refinement of a spatial search strategy after familiarization with task procedures, CIPL values for blocks 2,3 , and 4 were analyzed with a second repeated-measures ANOVA. This analysis revealed a significant main effect of block $\left(F_{(2,34)}=3.79, P<0.03\right)$. Moreover, when block 1 was excluded, aged rats also showed greater CIPL values and thus impaired search strategy relative to young rats, based on a significant main effect of age $\left(F_{(1,17)}=4.24, P<0.05\right)$. The interaction of block $\times$ age was not statistically significant $\left(F_{(2,34)}=1.87, P=0.17\right)$.

Spatial memory abilities were assessed with probe tests on days 2,4 , 6 , and 8 of the water maze paradigm (Gallagher et al. 1993; Bizon et al. 2009; McQuail and Nicolle 2015), in which the platform was lowered out of reach and rats were allowed to swim for 30 sec. Mean proximity to the learned platform location during each test was calculated and used as an index of targeted spatial search. Similar to the training trials, performance improved overall across successive probe tests (Fig. 1B), and there was a significant main effect of testing block $\left(F_{(3,51)}=7.36, P<0.001\right)$. Within subjects contrasts confirmed that overall, performance improved significantly from probe test 1 to tests 3 and 4 ( $P$ s $<$ $0.008)$. The ANOVA also revealed a significant main effect of age $\left(F_{(1,17)}=\right.$ 5.62, $P<0.03)$. Spatial learning index (SLI) values were compiled from probe tests given on days 4, 6, and 8 (Gallagher et al. 1993). SLI values of aged rats were significantly greater than those of young (Fig. 1C; $t_{(13.3)}=-3.09, P<0.008$; Levene's test: $F=7.48, P<0.01)$, reflecting a less targeted spatial search strategy in the aged group.

Following training and probe tests, rats underwent a cued test in which the escape platform remained visible above the water's surface, but was moved to a different location in the pool on each of six trials. Aged rats did not show evidence of visual, motor, or motivational deficits on these visible platform trials, as their mean path lengths taken to locate the visible platform did not differ from those of young rats (Fig. 1D; Mann-Whitney $U=33$, $P=0.33$; Shapiro-Wilk test: $P<0.002$ ).

\section{Spatial discrimination performance depends on the distance between target and foil locations}

To assess spatial discrimination abilities, we adapted a task in which rats distinguish between target and foil locations separated by variable distances on a hole-board maze (Gilbert et al. 1998, 2001; Kesner and Gilbert 2006). Performance of more difficult spatial discriminations on this task, when target and foil locations are moved closer together, has been shown to require the dentate gyrus (Gilbert et al. 2001). Rats were first trained on a match-toposition response strategy to retrieve a food reward from beneath one of two identical objects (Fig. 2A; Training Phase, also see Supplemental Fig. 1). Rats completed 16 trials per day in which objects were separated by a distance of 96 or $80 \mathrm{~cm}$, with eight trials of each spatial separation. Young rats acquired the matchto-position strategy within $138-804$ trials (range $=666$ trials; $10-51 \mathrm{~d}$ ) and aged rats within 114-1068 trials (range $=954$ trials; $7-67 \mathrm{~d})$. The number of trials required to reach criterion $(\geq 81.3 \%$ correct responses) was not influenced by age. Specifically, young and aged rats did not differ in the total number of training trials completed (Fig. 2C; Mann-Whitney $U=33, P=0.33$; ShapiroWilk test: $P<0.01$ ), or in the number of incorrect responses made prior to reaching criterion (Fig. 2D; Mann-Whitney $U=$ 32, $P=0.29$; Shapiro-Wilk test: $P<0.01$ ). Mean percent correct responses on the five training days prior to reaching criterion also did not differ between age groups (Fig. $2 \mathrm{E} ; t_{(17)}=-0.44$, $P=0.66)$.

After reaching criterion, a test of spatial discrimination was carried out over the following $5 \mathrm{~d}$ (Fig. 2B; Discrimination Test
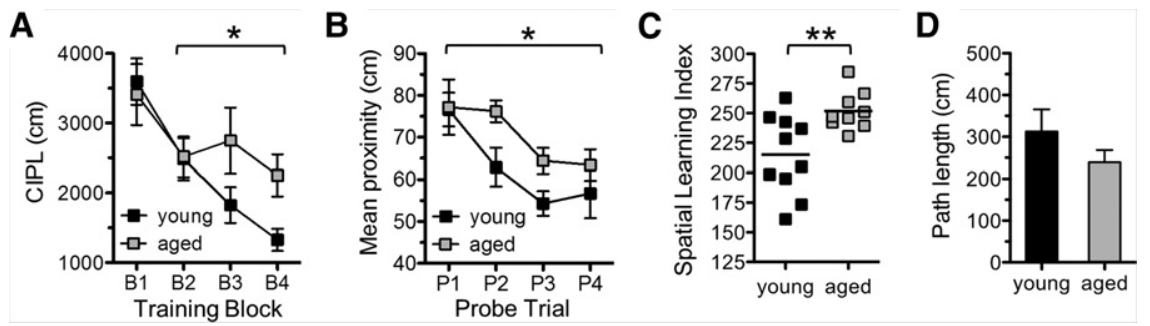

Figure 1. Aged rats show impairments in spatial memory on the Morris water maze task that are not attributable to visual, motor, or motivational deficits. (A) Cumulative integrated path length (CIPL) values reflecting the total distance traveled $(\mathrm{cm})$ prior to locating the hidden platform during water maze training, corrected for swim speed differences. All rats learned to locate the platform more effectively across training blocks $1-4$ (main effect of block: $P<0.001$ ), such that CIPL values for block 1 were greater than those for each other block (within subjects contrasts: $P S<0.01$ ). The decrease in CIPL from block 1 to block 2 likely reflects procedural learning, and did not differ based on age. However, young rats continued to refine their swim strategy from blocks 2 through 4, while aged did not (main effect of age: $P<0.05)$. (B) Mean proximity to the platform's target location during Probe tests 1 through 4. Proximity values decreased in both groups across probe tests (main effect of test: $P<0.0001$ ), but were greater overall in aged rats, reflecting poor memory for the target location (main effect of age: $P<0.03$ ). (C) Spatial learning index (SLI) values calculated for young and aged rats. Lines indicate group means. SLI values for aged rats were greater than those of young, reflecting spatial memory deficits $(P<0.008)$. $(D)$ Swim path lengths $(\mathrm{cm})$ of young and aged rats did not differ when the platform location was visible $(P=0.33)$. Graphs show mean \pm SEM. $(*) P<0.05\left({ }^{* *}\right) P<0.01$. 
Phase). On each daily test session, the distance between target and foil locations was varied to manipulate the difficulty of the discrimination. Rats completed 18 trials per day, with objects separated by a distance of 88,48 , or $15 \mathrm{~cm}$ (six trials of each). Spatial discrimination performance decreased on the more difficult trials, in which the target and foil locations were closer together (Fig. 2F). A repeated-measures ANOVA with distance between target and foil as a within subjects factor and age as a between subjects factor yielded a significant main effect of distance on percent correct responses $\left(F_{(2,34)}=21.4, P<0.001\right)$. Within subjects simple contrasts confirmed that rats made more correct responses on 88 -cm trials relative to 48 -cm trials $\left(F_{(1,17)}=7.86, P<0.01\right)$, and relative to 15 -cm trials $\left(F_{(1,17)}=41.7, P<0.001\right)$. The main effect of age $\left(F_{(1,17)}=3.17, P=0.09\right)$ and distance $\times$ age interaction $\left(F_{(2,34)}=0.29, P=0.75\right)$ were not statistically significant.

\section{Spatial discrimination performance in aged rats is vulnerable to cumulative interference}

In addition to differentiating between two cued spatial locations, performance on the spatial discrimination task requires distinction of just-cued locations from similar, recently cued locations. Given that one hallmark of cognitive aging is an increased susceptibility to interference (Hartman 1995; Pettigrew and Martin 2014; Wais and Gazzaley 2014), we considered the possibility that aged rats would show decreased performance within test sessions as they progress from early-to-late trials. We also hypothe- sized that switching from presentation of only easy trials during training to presentation of three levels of difficulty during test sessions would increase susceptibility to interference. To address this question, we collapsed performance across the final $5 \mathrm{~d}$ of training and the $5 \mathrm{~d}$ of discrimination tests, and compared percent correct responses during the first half of each session (early; Training trials 1-8, Test trials 1-9) and second half of each session (late; Training trials 9-16, Test trials 10-18) (Fig. 3A). A repeated-measures ANOVA comparing change in performance from early-to-late trials (\% correct late - \% correct early), with experimental phase as a within subjects factor (Training, Test) and age as a between subjects factor showed a significant main effect of phase $\left(F_{(1,17)}=5.31, P<0.03\right)$ and a significant phase $\times$ age interaction $\left(F_{(1,17)}=4.62, P<0.05\right)$. Post hoc contrasts between groups revealed that young and aged rats did not differ in the percent correct responses made on early test trials (Fig. 3A; $\left.F_{(1,17)}=0.71, P=0.41\right)$, but that, relative to young, aged rats made fewer correct responses on late test trials (Fig. $3 \mathrm{~A} ; F_{(1,17)}=$ 6.84, $P<0.02$ ).

To probe whether susceptibility to interference within test sessions was compounded by trial difficulty, correct responses for each level of difficulty were considered separately (Fig. 3B). Change in performance from early to late trials was calculated as described above, and these values were submitted to a repeated-measures ANOVA with distance as a within subjects factor $(88,48,15 \mathrm{~cm})$ and age as a between subjects factor. Main effects of distance $(P=0.93)$ and age $(P=0.09)$ and the
A

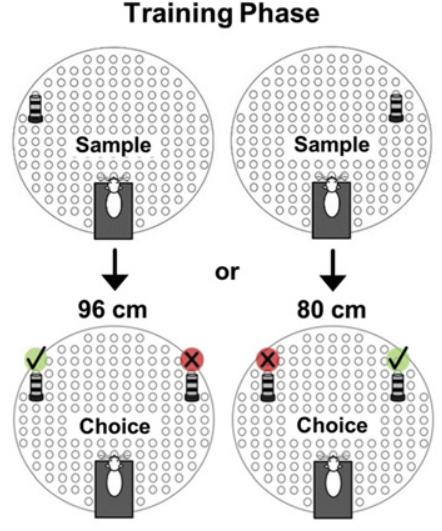

C

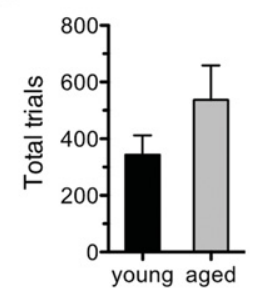

D

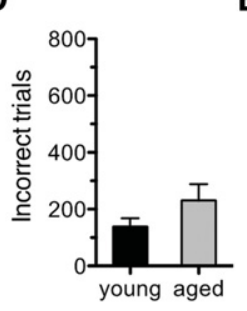

B

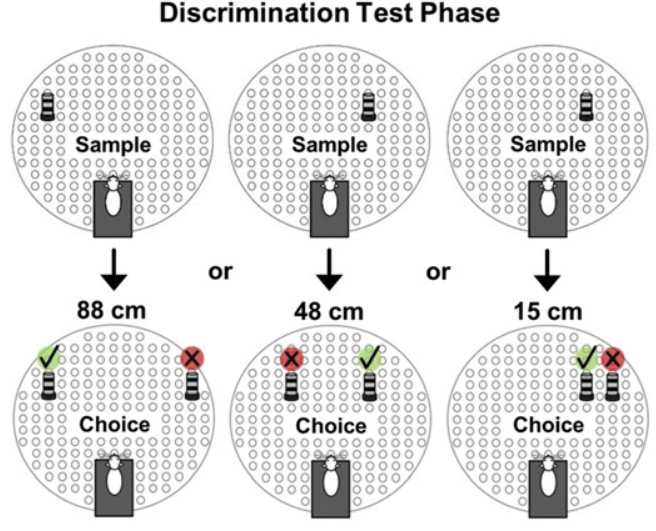

E

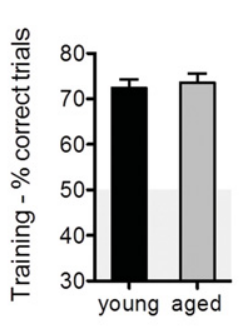

$\mathbf{F}$

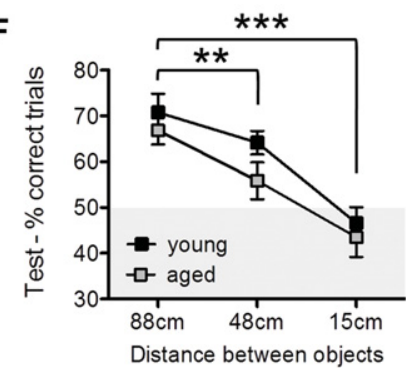

Figure 2. Spatial discrimination performance in young and aged rats. (A) Rats first learned a match-to-position response strategy (Training Phase). A specific food well was baited and cued with an object (Sample). After a brief delay, rats were presented with two identical copies of the same cue object separated by a distance of 96 or $80 \mathrm{~cm}$, covering the just-rewarded Target location and a Foil location (Choice). (B) Spatial discrimination performance was assessed by systematically varying the distance between Target and Foil locations (Discrimination Test Phase). (C,D) Young and aged rats acquired the match-to-position strategy within the same course of training. The two groups did not differ in their number of trials completed $(C ; P=0.33)$ or in number of incorrect responses made $(D ; P=0.29)$ prior to achieving criterion performance of $\geq 81.3 \%$ correct responses on two consecutive days. $(E)$ Young and aged did not differ in their spatial discrimination performance across the final $5 \mathrm{~d}$ of training, based on the percent correct responses made $(P=0.66)$. $(F)$ Spatial discrimination performance across easy $(88 \mathrm{~cm})$, difficult $(48 \mathrm{~cm})$, and very difficult $(15 \mathrm{~cm})$ spatial separations. Performance decreased significantly as a function of difficulty (main effect of distance: $P<0.001$ ), such that performance on $88-\mathrm{cm}$ trials was better than that on $48 \mathrm{~cm}$ or 15 -cm trials across age groups ( $P$ s $<0.01)$. Graphs show means \pm SEMs. $\left({ }^{* *}\right) P<0.01\left(^{* * *}\right) P<0.001$. 

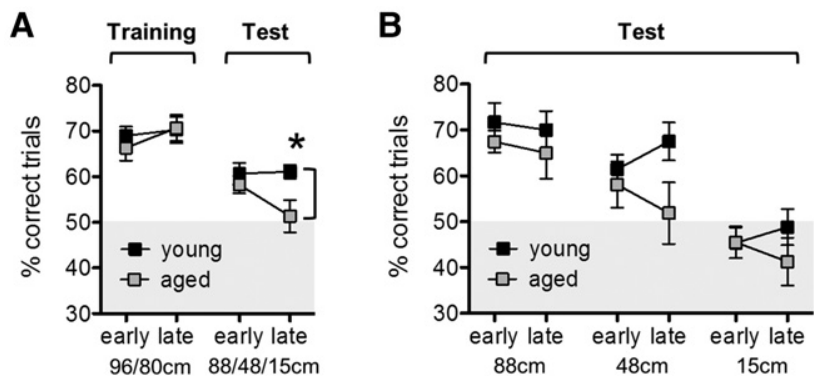

Figure 3. Aged rats showed a decrease in performance attributable to cumulative interference when task demand was highest, based on discrimination difficulty and the number of trial types presented. Trials were separated based on whether they took place in the first half (early) or second half (late) of training and test sessions. (A) Performance on early versus late trials collapsed across the last $5 \mathrm{~d}$ of the training phase (Training; $96 / 80 \mathrm{~cm}$ separations) and all $5 \mathrm{~d}$ of the discrimination test phase (Test; $88 / 48 / 15 \mathrm{~cm}$ separations). Change in performance from early to late trials differed from training to testing (main effect of phase: $P<0.03$ ), and was particularly apparent in aged rats during discrimination tests (phase $\times$ age interaction: $P<0.05$ ). On discrimination tests, young and aged rats did not differ in percent correct responses made on early trials $(P=0.41)$. However, aged rats were more prone to errors on late trials, showing significantly reduced performance relative to young $(P<0.02)$. (B) To determine whether aged rats' susceptibility to interference during late trials was modulated by trial difficulty, performance across early versus late trials was compared for each spatial separation. Repeated-measures ANOVA revealed a trend toward a significant main effect of age $(P=0.09)$. Graphs show means \pm SEMs. $\left(^{*}\right) P<0.05$.

distance $\times$ age interaction $(P=0.59)$ were not statistically significant. Our ability to detect statistical differences between age groups with this analysis was reduced by the small number of trials compared across each distance ( 3 trials $\times 5 \mathrm{~d}=15$ trials per cell), relative to the total number of trials compared in the overall analysis of early versus late performance on discrimination tests ( 9 trials collapsed across distance $\times 5 \mathrm{~d}=45$ trials per cell).

To further examine whether decreased performance of aged rats in late relative to early discrimination trials could be attributed to reduced motivation, fatigue, or other factors that selectively impact performance in the second half of test sessions, two experimental control phases were carried out. Rats first completed $2 \mathrm{~d}$ of discrimination tests with only $48-\mathrm{cm}$ trials (Supplemental Fig. 2A), followed by $2 \mathrm{~d}$ of $15-\mathrm{cm}$ trials (Supplemental Fig. 2D). Aged rats did not show overall impairments on either of these tests based on the distance between target and foil locations. Furthermore, rather than decreasing on late relative to early trials, performance generally improved in both young and aged rats (Supplemental Fig. 2C, F). These data confirm that aged rats' decreased performance on late trials during discrimination tests was unlikely to result from satiation or fatigue, and was contingent on task complexity, only emerging when rats completed multiple trial difficulties within test sessions.

\section{Spatial memory and spatial discrimination abilities are not correlated within individual rats}

Both spatial memory and spatial discrimination require intact hippocampal function (Schenk and Morris 1985; Xavier et al. 1999; Gilbert et al. 1998, 2001; Gilbert and Kesner 2006; Morris et al. 2012), and age-related differences in hippocampal-dependent memory function have recently been linked to differences in discrimination abilities in humans (Stark et al. 2013; Reagh et al. 2014, 2016), as well as in rats (LaSarge et al. 2007). We therefore sought to determine whether spatial memory performance on the water maze task was correlated with spatial discrimination performance across our cohort of young and aged rats. Variables of interest were normalized and correlational analyses were carried out on the resulting $Z$-scores. In the young group, young rats with better spatial memory (i.e., lower SLI values) tended to have poor spatial discrimination abilities (i.e., fewer correct responses on $48-\mathrm{cm}$ trials), although this correlation did not reach statistical significance (Fig. $4 \mathrm{~A} ; R=0.613, P=0.06$ ). In the aged group, SLI values and discrimination performance on $48-\mathrm{cm}$ trials were not significantly correlated (Fig. 4B; $R=-0.305, P=0.43$ ). SLI values were also not correlated with discrimination performance during the training phase or performance on $88-\mathrm{cm}$ trials during the test phase, across groups or when young and aged were considered separately $(P s>0.11)$. Plotting the performance of individual rats on $48 \mathrm{~cm}$ discrimination trials (Fig. 4C) revealed that two of the nine aged rats showed particularly superior abilities $(73.3 \%$ correct responses), such that their performance on these trials fell above the normative range of both the young and aged groups (young Mean $=64.2 \%, \mathrm{SD}=8.1$, Mean +1 $\mathrm{SD}=72.3 \%$; aged Mean $=55.8 \%, \mathrm{SD}=12.1$, Mean $+1 \mathrm{SD}=$ $67.9 \%$ ). Exclusion of these two high-performing rats from the aged group revealed a significant impairment in discrimination abilities relative to young in the remaining seven aged rats $\left(t_{(15)}=3.36, P<0.004\right)$, but did not reveal a correlation between SLI values and performance on $48 \mathrm{~cm}$ discrimination trials in this subset of aged animals $(R=0.023, P=0.96)$. Taken together, these analyses indicate that with the experimental procedures used, individual rats' spatial memory abilities do not predict their spatial discrimination abilities. The lack of correlation between these two variables suggests our implementations of the water maze and hole-board discrimination task may tax distinct neural circuits, both in young and aged rats.

\section{Spatial discrimination performance after systemic donepezil injections}

Cholinergic neurotransmission is critical to attention and memory (Hasselmo 2006), and can influence abilities to discriminate between similar stimuli in other sensory domains (Devore et al. 2014). To determine whether increasing acetylcholine levels would alter spatial discrimination performance, rats received intraperitoneal injections of the acetylcholinesterase inhibitor donepezil ( 1 and $3 \mathrm{mg} / \mathrm{kg}$ ) or vehicle prior to discrimination tests. Performance was initially compared across all injection conditions, but revealed no significant effect of the higher donepezil dose. Considering systemic treatment with cholinergic agonists at high doses can be associated with peripheral side effects (Jackson et al. 2004), and that we occasionally observed behavioral signs of malaise in both young and aged rats following high dose injections, we excluded these data from subsequent analyses.

To determine whether donepezil improved spatial discrimination performance, change in percent correct responses based on injection condition was calculated for each individual rat for 88- and 48-cm trials (\% correct after donepezil $1 \mathrm{mg} / \mathrm{kg}-\%$ correct after vehicle). Data from $15-\mathrm{cm}$ trials were excluded from analyses because young and aged groups continued to perform at chance across injection conditions. Donepezil's effects on performance were examined with a repeated-measures ANOVA, with distance between target and foil locations $(88,48 \mathrm{~cm})$ as a withinsubjects factor and age as a between subjects factor. Donepezil injections appeared to differentially affect performance in young and aged rats; however, the main effect of age (Fig. $5 \mathrm{~A} ; F_{(1,15)}=$ 3.11, $P=0.09)$ and distance $\times$ age interaction $\left(F_{(1,15)}=3.46, P=\right.$ 0.08 ) were not statistically significant. Post hoc contrasts revealed no difference between age groups in donepezil's effects on $88-\mathrm{cm}$ trials $\left(F_{(1,15)}=0.24, P=0.63\right)$, as both young and aged showed slight improvement from vehicle to donepezil conditions (young Mean $=+3.9 \%, \mathrm{SD}=6.98$; aged Mean $=+6.75 \%, \mathrm{SD}=16.5$ ). 
A

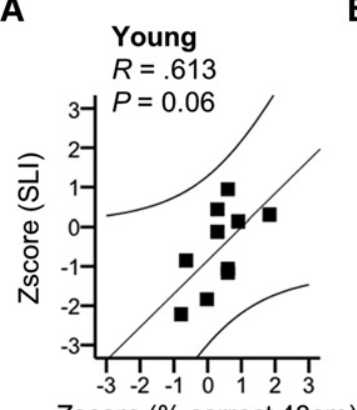

Zscore (\% correct $48 \mathrm{~cm})$
B

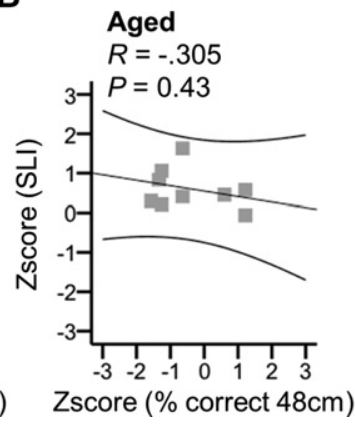

C

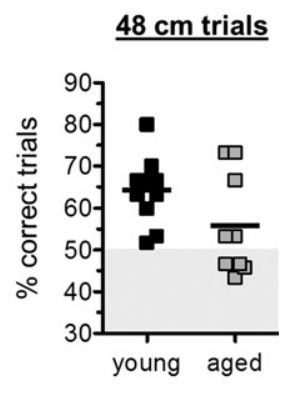

Figure 4. Better spatial memory is not associated with better spatial discrimination abilities in young or aged rats. SLI values and percent correct responses on $48-\mathrm{cm}$ trials were normalized and correlations were computed separately for young and aged groups. $(A)$ In the young group, a statistical trend toward a positive correlation indicated young rats with better spatial memory (i.e., lower SLI values) were more likely to have poor spatial discrimination abilities (i.e., fewer correct responses on $48-\mathrm{cm}$ trials) $(R=0.613, P=0.06)$. (B) In the aged group, SLI values and discrimination performance on $48-\mathrm{cm}$ trials were not significantly correlated $(R=-0.305, P=0.43)$. (C) Individual rats' performance on 48-cm trials during tests for spatial discrimination abilities. Horizontal bars indicate group means. Two of the nine aged rats showed superior discrimination abilities on these trials (i.e., $73.3 \%$ correct responses). Performance of these two rats was above the normative range of both young and aged groups (young Mean $=64.2 \%, S D=8.1$, Mean $+1 S D=72.3 \%$; aged Mean $=55.8 \%, S D=12.1$, Mean $+1 S D=67.9 \%)$. Scatterplots show best fit line $\pm 95 \%$ confidence intervals.

Donepezil differentially influenced performance on 48 -cm trials in young and aged rats $\left(F_{(1,15)}=4.87, P<0.04\right)$. Aged rats showed improvement following donepezil relative to vehicle injections (Mean $=+9.14 \%, \mathrm{SD}=13.5)$, while young rats showed decreased performance attributable to donepezil (Mean $=-8.04 \%, \mathrm{SD}=$ 17.2). Comparison of individual rats' performance on $48-\mathrm{cm}$ trials revealed that a subset of young rats showed a decline in percent correct responses after donepezil relative to vehicle injections (Supplemental Fig. 3A), while aged rats were more likely to show greater percent correct responses on these trials after donepezil relative to vehicle (Supplemental Fig. 3B).

To determine whether donepezil reduced the effects of cumulative interference in aged rats, performance on early and late trials were entered into two repeated-measures ANOVAs with drug condition as a within-subjects factor (vehicle, donepezil $1 \mathrm{mg} / \mathrm{kg}$ ) and age as a between subjects factor. These analyses did not reveal significant main effects of drug or drug $\times$ age interactions (Fig. 5B; Ps $>0.20$ ). However, there were significant main effects of age on early trials $\left(F_{(1,15)}=4.49, P<0.05\right)$ and late trials similar pattern (Supplemental Fig. 3). However, a $t$-test comparing overall change in performance after donepezil in young versus aged animals was not statistically significant (Fig. 5C; $t_{(13)}=-1.77, P=0.09$ ).

\section{Discussion}

In these experiments we document several novel observations regarding the impact of aging on spatial discrimination, as well as the relationship between spatial discrimination and spatial memory abilities as tested on the Morris water maze task. First, a majority of aged rats were impaired on spatial discriminations in which the distance between target and foil locations was $<88 \mathrm{~cm}$. These data support an emerging consensus in the field of cognitive aging that age-associated discrimination deficits in humans and animal models are observed when the test stimuli are similar or share common features (Stark et al. 2010, 2013; Burke et al. 2011; Yassa et al. 2011a,b; Ryan et al. 2012; Holden et al. 2013; Reagh et al. 2014, 2016). Moreover, the observation that a subset of aged rats were
A

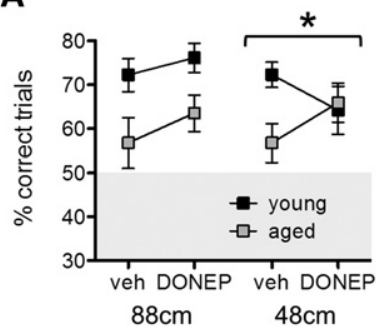

B

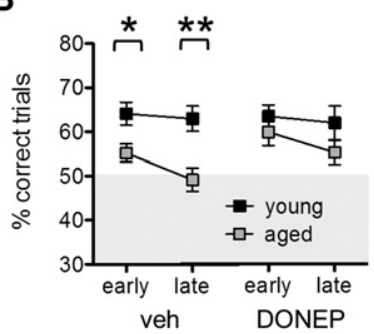

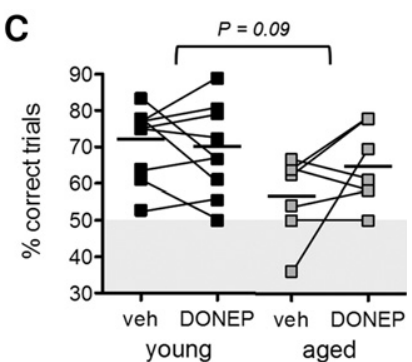

Figure 5. Spatial discrimination performance after systemic donepezil injections. ( $A$ ) No age difference was present in the effect of donepezil injections on 88-cm trials $(P=0.63)$; however, donepezil differentially influenced performance of young and aged rats on $48-\mathrm{cm}$ trials $(P<0.04)$. Aged rats showed improvement with donepezil (mean change from vehicle $=+9.14 \%, S D=13.5$ ), while young rats showed decreased performance (mean change from vehicle $=-8.04 \%, S D=17.2$ ). Data from $15-\mathrm{cm}$ trials were excluded from analyses because performance remained consistently at chance across all experimental conditions. $(B)$ Performance on easy versus late discrimination trials after vehicle or donepezil injections. Donepezil improved performance across both early and late phases in aged rats such that significant impairments observed after vehicle injections $(P s<0.02)$ were alleviated $(P s>$ 0.21). (C) To test whether donepezil had a more general cognitive-enhancing effect, percent correct responses were collapsed across 88-cm and 48-cm trials. Individual performance is shown for young rats (left; black markers) and aged rats (right; gray markers); horizontal lines indicate group means. A statistical trend indicated that, overall, aged rats tended to benefit from donepezil treatment (mean change from vehicle $=+7.9 \%$, SD $=$ 14) while young rats did not (mean change from vehicle $=-2.1 \%, \mathrm{SD}=9.5 ; P=0.09$ ). Graphs show means $\pm \operatorname{SEMs} .\left(^{*}\right) P<0.05\left({ }^{* *}\right) P<0.01$. 
unimpaired in performing difficult spatial discriminations parallels recent data from aged humans, showing spatial discrimination abilities are less prone to decline with aging than visual object discrimination abilities (Reagh et al. 2016). Future experiments will need to cross-characterize animals on object and spatial discrimination problems and directly measure the extent to which these modalities are differentially vulnerable in aged rats.

The current experiments also show that aged rats' performance on late relative to early discrimination trials was prone to interference. To our knowledge, this is the first report of a susceptibility to cumulative interference in aged rats. This result suggests that, when faced with difficult discriminations after an increasing number of trials, aged rats default to previously stored representations. Perhaps counterintuitively, our results also revealed that spatial memory performance on the Morris water maze, in which effects of age are well-documented, does not predict spatial discrimination performance. This may be a factor of strain differences in water maze performance in both young and aged rats, which is discussed in more detail below. Furthermore, treatment with the acetylcholinesterase inhibitor donepezil was generally without effect on spatial discrimination abilities, yet improved performance in a subset of aged rats across all trials without selectively ameliorating difficulty- or interference-dependent deficits.

\section{Spatial memory and spatial discrimination abilities: implications for the neural substrates of discrimination}

In the current study, the majority of aged rats tested were impaired on difficult spatial discriminations (Fig. 4C). Our results build on a similar finding that aged rats made more errors in a radial arm maze test of spatial working memory when there was a high degree of overlap among environmental cues associated with target and foil arms (Gracian et al. 2013). The discrimination task we used has been previously shown to rely on hippocampal function (Gilbert et al. 1998), and more specifically on the dentate gyrus (Gilbert et al. 2001). Furthermore, dentate gyrus lesions in young rats impair performance on spatial working memory tests (Xavier et al. 1999; Morris et al. 2012). Thus, lesion studies in rats suggest that age-related deficits in discriminating between spatial stimuli could be linked to age-related changes in hippocampal function, and particularly to the dentate gyrus.

In the present study, however, no relationship was detected between spatial discrimination and water maze performance. Despite evidence that both tasks require hippocampal function, there are a number of potential reasons for this lack of correlation. First, the distribution of SLI values for our young and aged groups differed from that typically observed in young and aged LongEvans (Gallagher et al. 1993) or Fischer 344 rats (LaSarge et al. 2007; Bizon et al. 2009). This is not necessarily surprising, as the F344 $\times$ BN hybrid rats have been found to show differences in behavioral variables across multiple cognitive tasks, including the water maze and other tests of spatial and contextual memory (Spangler et al. 1994; van der Staay and Blokland 1996). Nevertheless, a subset of our young rats showed relatively poor spatial memory based on SLI values, while none of our aged rats had relatively good spatial memory based on SLI values (cf. McQuail and Nicolle 2015). This underscores the fact that SLI values from one laboratory are not necessarily comparable to those from another, even when the rat strain, water maze apparatus, and experimental protocol used are identical (McQuail and Nicolle 2015). It is possible that subtle variations in the experimental setup, including the prominence and distribution of extra-maze cues, biased rats toward using a less spatial and more response-based strategy during probe trials (Tomás Pereira et al. 2015).

Another potential explanation for the lack of relationship between water maze performance and spatial discrimination is that these tasks may rely on distinct extra-hippocampal structures. For example, frontal and parietal cortical regions are required for successful water maze performance (DiMattia and Kesner 1988; de Bruin et al. 1994; Vafaei and Rashidy-Pour 2004). It is therefore possible that age-related deficits in spatial memory arise not only from well-documented hippocampal dysfunction (Burke and Barnes 2006; Gray and Barnes 2015), but also in part from changes in frontal cortical regions or hippocampal-frontal connectivity. Nonetheless, one notable difference between the spatial discrimination task and the water maze task as implemented in the present study is that the goal location in the water maze remains fixed across all training and test trials, while the target location in the spatial discrimination task changes from trial to trial. This requirement of rats to maintain a location in memory for a brief period and then update that location across trials likely engages prefrontal cortical circuits (Sloan et al. 2006). Because prefrontal cortical function is also vulnerable to the effects of advanced age (Morrison and Baxter 2012; Beas et al. 2013, 2016; Bañuelos et al. 2014), deficits in spatial discrimination performance could potentially emerge from dysfunction of this brain area or its ability to interact with medial temporal lobe structures.

In contrast to animal studies, the majority of discrimination studies in humans have used pictures of common objects as stimuli (Toner et al. 2009; Yassa et al. 2011a,b; Holden et al. 2013; Stark et al. 2013; Reagh et al. 2014, 2016). Performing these tasks therefore presumably draws on brain networks required for object processing, such as the perirhinal cortex. Of note, human studies that have assessed spatial discrimination have also used screen-based presentation of stimuli, requiring subjects to gauge the position of shapes or pictures of objects on the screen (Stark et al. 2010; Holden et al. 2012; Reagh et al. 2014, 2016), which is quite different from using real world spatial representations and/or path integration to guide discrimination. Nonetheless, studies using this type of stimulus presentation have found age-associated discrimination deficits to be correlated with altered CA3 and dentate gyrus activity (Yassa et al. 2011a,b; Bakker et al. 2012), and more specifically with loss of integrity of perforant path inputs from the entorhinal cortex to the hippocampus and dentate gyrus (Bennett et al. 2015; Bennett and Stark 2016). The extent to which prefrontal cortical input is also necessary and engaged by these behaviors in humans will need to be explored.

A question that remains open is whether brain systems required for object processing are also necessary for discrimination of spatial information, and by consequence, whether age-related dysfunction of these systems (Burke et al. 2012, 2014; Ryan et al. 2012) contributes to memory loss that is often solely attributed to the hippocampus. Along these lines, age-related deficits on object-based discrimination tasks (Toner et al. 2009; Yassa et al. 2011a,b; Stark et al. 2013; Reagh et al. 2016) are reminiscent of the reduced ability of rats (Bartko et al. 2007a,b) and monkeys (Bussey et al. 2002, 2003) to discriminate between similar stimuli following perirhinal cortical lesions. In fact, one study to date in humans has linked age-related impairments in difficult object discriminations with perirhinal cortex activity (Ryan et al. 2012). Although perirhinal cortical lesions can lead to deficits in spatial working memory (Wiig and Burwell 1998), the contribution of cortical-hippocampal connectivity to age-related deficits in spatial discrimination ability has not been directly tested and should be the focus of future studies in both human subjects and animal models.

\section{The role of interference in spatial discrimination deficits} The current study also revealed that age-related deficits on spatial discrimination tests were primarily due to aged rats' declining performance on late relative to early trials. We attribute decrements 
in performance on late trials to a greater potential for cumulative interference as trials pass and representations of previous goal locations accumulate. This interpretation is consistent with recent data showing older adults are impaired in recalling stimulus locations when similar or identical stimuli were recently presented (Sapkota et al. 2014). Our finding also suggests the spatial discrimination test used in the current study taxes executive functions by requiring rats to store information regarding spatial locations for a single trial and then update that information across trials. Consistent with this observation, both working memory (Moss et al. 2007; Beas et al. 2013; Bañuelos et al. 2014) and set-shifting (Moore et al. 2003, 2006; Beas et al. 2013, 2016; Bañuelos et al. 2014) are impaired by aging.

The possibility that working memory is increasingly required in aged individuals when stimuli share overlapping features is supported by several lines of evidence from studies in humans. Relative to young adults, older subjects are particularly susceptible to proactive interference on working memory tests when similar stimuli are presented (Lustig and Hasher 2001; Rowe et al. 2010). Older adults also show a reduced ability to suppress irrelevant information and are more likely to take this information into account when selecting responses, an effect that is prominent when stimuli were experienced in temporal proximity or within the same sensory modality (Gazzaley et al. 2005; Campbell et al. 2014). Given that the match-to-position task implemented in the current study requires resolution of similar visuospatial stimuli and active suppression of irrelevant information, since goal locations from previous trials are no longer useful for present trials, it is probable that working memory abilities contribute to successful performance. By consequence, the spatial discrimination task likely relies on both the hippocampus and prefrontal cortex and in particular on the connectivity between these structures. While the effects of aging on prefrontal cortical functions have been well-characterized in the context of both episodic and working memory (Rajah and D'Esposito 2005; Morrison and Baxter 2012), contributions of the prefrontal cortex to discrimination ability have not yet been examined.

\section{Cholinergic function and spatial discrimination}

We found that increasing acetylcholine levels did not selectively reverse the difficulty- or interference-dependent effects of aging on spatial discrimination performance. This suggests that donepezil acted on a more general cognitive process that lent itself to improving overall performance, but did not target neurobiological substrates specifically underlying spatial discrimination abilities. Furthermore, the heterogeneity of donepezil's effects across individual rats (Fig. 4C; Supplemental Fig. 3) supports the interpretation that differences in baseline cholinergic function influenced the relative effects of the drug on cognitive function in both young and aged groups. In line with this conclusion, we found that a subset of young rats showed decreased performance on 48-cm trials after receiving donepezil injections (see Supplemental Fig. 3A). Given that peripheral side effects of donepezil have been documented in clinical populations (Jackson et al. 2004), it is possible that young rats with higher endogenous levels of acetylcholine were negatively affected by the drug.

Another factor that may have limited our ability to detect a pro-cognitive effect of donepezil in the present study is the fact that aged rats showed a drop in performance from the initial discrimination test phase to the pharmacology test phase (see Figs. $2 \mathrm{~F}, 5 \mathrm{~A}$ ). This decrease likely arose from the duration of intervening control experiments (as documented in Supplemental Fig. 2), and from the reduced practice available when rats were tested every $3 \mathrm{~d}$ rather than every day to allow for the within-subjects design of the pharmacology study. Given the aged group began the study at 24 $\mathrm{m}$ of age, it is also conceivable that additional cognitive decline took place prior to completing the pharmacology experiment and then over the course of the pharmacology experiment. It is possible that pharmacological intervention earlier in the lifespan, or with a more consistent daily discrimination test regimen, would have been more effective in enhancing difficult discrimination abilities or reducing the potential for cumulative interference. Nevertheless, it is worth noting that donepezil substantially improved performance in a subset of the aged rats despite these factors.

Prior studies in aged rats have found that chronic treatment with donepezil did not affect spatial working memory (Barnes et al. 2000), but improved spatial reference memory (Hernandez et al. 2006). Similarly, older adults diagnosed with mild cognitive impairment who were treated with galantamine showed improvements in delayed recall, but not measures of attention, working memory, or susceptibility to interference (Grön et al. 2006). While these findings may be inconsistent with the established role of cholinergic function in sustained attention and working memory (Sarter and Bruno 1997), it is possible that with the doses and time courses used, including those of the present experiments, cholinesterase inhibitors lack the neurobiological specificity to improve attentional and working memory performance in aged individuals.

\section{Conclusions}

The results of this study demonstrate for the first time that aged rats can be impaired in spatial discrimination relative to young adult rats. Our finding closely parallels results from human studies (Stark et al. 2013; Reagh et al. 2014, 2016), and adds to a growing body of data that indicates discrimination of complex stimuli can be a sensitive behavioral index of cognitive aging, perhaps even more so than standard neuropsychological measures assessing delay-dependent memory. We describe this cognitive ability and behavioral measure as "discrimination" to avoid confusion with "pattern separation". Though in recent years the latter term has been adopted by many, including us (Burke et al. 2010, 2011), to refer to behavioral discrimination performance, it is important in moving forward to disambiguate behavioral measures of discrimination from the computational process of pattern separation, as described by Marr, Rolls, and others (Marr 1971; McNaughton and Morris 1987; Treves and Rolls 1992; O'Reilly and Norman 2002; Rolls and Kesner 2006; Santoro 2013; Kesner and Rolls 2015). With this distinction in mind, continued implementation and refinement of difficulty-dependent discrimination tasks in rodents may shed light on the effects of aging on these measures as well as lend itself to empirical studies testing the neurobiological bases of pattern separation in terms of computational processes across hippocampal and cortical circuits.

\section{Materials and Methods}

\section{Animals}

A total of 11 young adult $(4 \mathrm{~m})$ and 12 aged $(24 \mathrm{~m})$ male Fischer $344 \times$ Brown Norway F1 hybrid rats from the NIA colony (Taconic) were used in the present study. Of the 12 aged rats, one died of natural causes and two were killed after developing complications associated with aging during spatial discrimination training. Further, one young rat showed aberrant performance on cued water maze trials relative to the young group in the present experimental cohort and the overall population of young rats screened using the same protocol in our laboratory $(N=40$ rats). As a result, 10 young and nine aged rats were compared in analyses of spatial memory and discrimination abilities. Two additional aged rats were removed for health reasons prior to beginning the pharmacology study; 11 young and seven aged rats 
were included in these analyses. Rats were housed individually in standard Plexiglas cages and maintained on a 12-h reversed lightdark cycle (lights off at 8:00 a.m.). All behavioral manipulations were performed during the dark phase of the cycle. Following assessment of spatial memory on the water maze, food access was restricted and rats were maintained at $85 \%$ of their initial freefeeding weights. Moist chow (Teklad LM-485, Harlan Labs) was provided once daily and access to water was provided ad libitum. All procedures were performed in accordance with National Institutes of Health guidelines and were approved by the Institutional Animal Care and Use Committee at the University of Florida.

\section{Water maze task}

Rats were tested on a spatial memory version of the Morris water maze task following the procedure of Bizon and colleagues (Gallagher et al. 1993; LaSarge et al. 2007; Bizon et al. 2009; McQuail and Nicolle 2015). The water maze consisted of a circular pool (1.83-m diameter, 58 -cm height) surrounded by black curtains to which visible landmark cues were attached. A hydraulic submersible platform (12-cm diameter; HVS Image) was positioned in one quadrant and remained in this fixed location. The pool was filled with water $\left(\sim 25^{\circ} \mathrm{C}\right)$ to a depth of 2 -cm above the platform, and white nontoxic tempera paint was added to obscure the platform's location. Position within the maze, path length, and swim speed were monitored and then computed across trials with a video-tracking system (Water 2100, HVS Image). Rats were acclimated to experimental handling and the water maze room prior to beginning the task. Three swim trials were administered each day for eight consecutive days, with probe tests conducted on the third swim trial of days $2,4,6$, and 8 . For each swim trial, rats were placed in the water facing the wall of the pool at one of four start positions, which were varied in a pseudorandom fashion such that all rats were required to locate the platform from each of the locations the same number of times. Rats were allowed a maximum of $90 \mathrm{sec}$ to locate the platform, after which the experimenter would guide the rat to the platform. Mean cumulative integrated path length (CIPL) values were calculated across each block of five training trials to provide an index of spatial learning. The CIPL is equivalent to the cumulative search error originally reported by Gallagher et al. (1993). These values reflect the total distance traveled in locating the platform on each training trial, corrected for the distance from each trial's drop-off point at the perimeter of the pool to the platform's target location (i.e., optimal path) and for each individual rat's swim speed. For probe tests, the platform was lowered 20-cm below the water's surface for the first $30 \mathrm{sec}$ and performance was quantified by measuring the proximity of each rat's swim path to the target location during the 30-sec probe test. Proximity values were corrected for swim speed and optimal swim path from drop-off point to the target location, as previously reported (Gallagher et al. 1993; LaSarge et al. 2007; Bizon et al. 2009; McQuail and Nicolle 2015). The spatial learning index (SLI) was computed from proximity values for probe tests administered on days 4,6 , and 8 of the water maze paradigm, as previously reported (Gallagher et al. 1993). Proximity values for the first probe test administered on day 2 of the water maze paradigm did not differ between young and aged groups (see Fig. 1B). The SLI is the weighted sum of proximity values from probe tests 2,3 , and 4 , with weightings derived based on the improvement in search strategy observed in a population of young adult animals across successive tests. For the present study, weightings were derived from a population of $N=40$ young adult F344 $\times$ BN hybrid rats that have now been characterized using the same protocol in our laboratory. Each weighting corresponds to the ratio of young rats' mean performance on probe tests 2,3 , or 4 (P2, P3, P4), relative to performance on probe test 1 (P1). For example, the weighting for P2 would be calculated as the ratio of mean proximity of young rats on P2 (Mean $=71.6 \mathrm{~cm})$ and mean proximity of young rats on P1 (Mean $=81.8$; Weight for $\mathrm{P} 2=81.8 / 71.6=1.14$ ). With these calculations based on our population of young adult rats, SLI values were calculated as follows: $\mathrm{SLI}=\mathrm{P} 2 \times 1.14+\mathrm{P} 3 \times 1.25+\mathrm{P} 4 \times 1.33$. These weightings were comparable to those previously derived for the $\mathrm{F} 344 \times \mathrm{BN}$ strain (McQuail and Nicolle 2015). Greater SLI values reflect greater search error, and thus worse performance.

On the day after completing the spatial memory task, rats were given a cued test as a control for visual impairment, sensorimotor impairment, or poor motivation that could influence performance. The water level was lowered to $2-\mathrm{cm}$ below the platform and a black ring was placed on the top of the platform to increase its visibility. The platform was moved to a different quadrant of the maze for each trial in a standard pattern, such that all rats were required to swim to the same platform locations from the same start positions. As for previous trials, rats were given $90 \mathrm{sec}$ to reach the platform and were allowed to remain there briefly before a 30-sec intertrial interval. Mean path length values across the cued trials were calculated as an index of swimming ability.

\section{Spatial discrimination training and testing}

After completing the water maze task, rats were placed on a restricted feeding regimen and began shaping for the spatial discrimination task, which is based on a task previously developed by Gilbert et al. (1998, 2001). Training and testing were carried out on a black circular hole-board maze (1.22-m diameter). A start box $(30 \mathrm{~cm}$ length $\times 20 \mathrm{~cm}$ width $\times 28 \mathrm{~cm}$ height $)$ was located on the south end of the maze and prominent visual cues were affixed to the walls (see Supplemental Fig. 1A for additional detail on apparatus and shaping procedure). Rats were initially trained on a match-to-position response strategy with easy spatial discriminations. Training trials consisted of two phases: Sample and Choice (Fig. 2A). During the Sample phase, a food well was baited with a small piece of Froot Loop cereal (Kellogg's) and a cue object distinct from that used during shaping was placed covering the food well (Supplemental Fig. 1C). Food wells baited for training and testing were always located within a designated row on the cheeseboard maze (Supplemental Fig. 1D). To initiate the Sample phase in each trial, rats were placed on the maze facing toward the start box. Rats were then allowed to freely explore the maze to locate and displace the cue object. Once they had retrieved the Froot Loop, rats were allowed a few seconds to consume the food reward in the Sample location. The experimenter then returned the rat to the start box for a brief $10-15$-sec intratrial delay and quickly rebaited the same food well, covering this well (Target) and a second well (Foil) with identical copies of the cue object (Fig. 2A; Supplemental Fig. 1C). To initiate the Choice phase of the trial, rats were placed on the maze facing away from the start box. Rats were again allowed to freely explore and choose between displacing the object covering the Target or Foil location. Once the rat displaced one of the objects, the experimenter returned the rat to the start box for a 45-60-sec intertrial delay. During training, Target and Foil locations were always separated by a distance of 96 or $80 \mathrm{~cm}$ to minimize overlap between environmental cues guiding response during the Choice phase (Fig. 2A). Each day, rats completed 16 trials, with 8 trials of each spatial separation. Rewarded side (L versus R) and spatial separation were pseudorandomly distributed and counterbalanced within training sessions and across training days. Discrimination testing began once rats reached criterion of 13 or more correct responses $(\geq 81.3 \%)$ on two consecutive days.

Discrimination testing took place over five consecutive days. Each day, rats were required to complete 18 trials using the match-to-position response strategy. To parametrically vary the difficulty of the spatial discrimination trials, Target and Foil locations during the Choice phase were now separated by a distance of 88 , 48, or $15 \mathrm{~cm}$ (Fig. 2B). Six trials of each spatial separation were pseudorandomly distributed within each test day, counterbalancing the side of the rewarded food well (L versus $\mathrm{R}$ ) and the spatial position of the objects within the designated row (i.e., both toward L, centered, or both toward R). To determine whether performance on the more difficult spatial discriminations would improve with practice, and to verify whether decreased performance of aged rats across trials might be the result of declines in motivation or fatigue, controls were then carried out in which rats completed $2 \mathrm{~d}$ of only $48 \mathrm{~cm}$ spatial separations (18 trials/ day) and $2 \mathrm{~d}$ of primarily $15-\mathrm{cm}$ separations (14 trials per day 
with 2 trials each of 48 and $88 \mathrm{~cm}$ separations randomly interleaved). Results of these experimental control phases are shown in Supplemental Figure 2.

\section{Effect of donepezil on spatial discrimination performance}

To determine whether enhancing cholinergic neurotransmission would improve spatial discrimination performance, rats were injected systemically with donepezil $\mathrm{HCl}(1 \mathrm{mg} / \mathrm{kg}$ or $3 \mathrm{mg} / \mathrm{kg}$ i.p.; USP) or vehicle (0.9\% physiological saline, $1 \mathrm{~mL} / \mathrm{kg}$ i.p.) 30 min prior to completing spatial discrimination tests described above. Injections and testing were carried out within subjects over three blocks, in which each rat received injections of vehicle, donepezil $1 \mathrm{mg} / \mathrm{kg}$, and donepezil $3 \mathrm{mg} / \mathrm{kg}$ on different days. Each injection per test day was followed by a 72-h wash out period, such that a full block would span $9 \mathrm{~d}$. The order in which vehicle and drug doses were administered was randomized within and across blocks for each rat. The number of correct responses made on test days under each dose were then collapsed and analyzed based on spatial separation $(88,48$, or $15 \mathrm{~cm})$.

\section{Statistical analyses}

Data are presented as mean values \pm SEMs. Analyses were performed with the Statistical Package for the Social Sciences (SPSS) v23 for Mac OS X. Behavioral variables were compared with repeated-measures ANOVAs, $t$-tests, or nonparametric MannWhitney $U$-tests, with experimental phase, spatial separation, or injection condition as within subjects factors and age as a between subjects factor. Choice of statistical test was dictated by assumptions of normality, assessed with Shapiro-Wilk tests, and homogeneity of variances, assessed with Levene's tests. Results of these tests are stated where applicable. Post hoc analyses were performed with simple contrasts where appropriate, with a Bonferroni correction applied. Relationships between spatial memory and discrimination abilities were determined with Pearson's correlations on normalized variables. $P$-values $<0.05$ were considered statistically significant.

\section{Acknowledgments}

We thank Katelyn Carty, Drew Fantaccione, Jack Morgan Mizell, Lindsay Santacroce, Leila Shafiq, Wembley Vilela, Ethan Windham, and Wendy Yoder for assistance in conducting behavioral experiments. We also thank Michael G. Burke for constructing the hole-board maze. This work was carried out with funds from the McKnight Brain Research Foundation, National Institute on Aging (R03 AG 049411), UF Research Seed Opportunity Fund, UF University Scholars Program, Brazil Scientific Mobility Program, and the UF HHMI Science for Life Program.

\section{References}

Bakker A, Krauss GL, Albert MS, Speck CL, Jones LR, Stark CE, Yassa MA, Bassett SS, Shelton AL, Gallagher M. 2012. Reduction of hippocampal hyperactivity improves cognition in amnestic mild cognitive impairment. Neuron 74: 467-474.

Bañuelos C, Beas BS, McQuail JA, Gilbert RJ, Frazier CJ, Setlow B, Bizon JL. 2014. Prefrontal cortical GABAergic dysfunction contributes to age-related working memory impairment. J Neurosci 34: 3457-3466.

Barnes CA, Rao G, McNaughton BL. 1996. Functional integrity of NMDA-dependent LTP induction mechanisms across the lifespan of F-344 rats. Learn Mem 3: 124-137.

Barnes CA, Meltzer J, Houston F, Orr G, McGann K, Wenk GL. 2000. Chronic treatment of old rats with donepezil or galantamine: effects on memory, hippocampal plasticity and nicotinic receptors. Neuroscience 99: $17-23$.

Bartko SJ, Winters BD, Cowell RA, Saksida LM, Bussey TJ. 2007a. Perceptual functions of perirhinal cortex in rats: zero-delay object recognition and simultaneous oddity discriminations. J Neurosci 27: 2548-2559.

Bartko SJ, Winters BD, Cowell RA, Saksida LM, Bussey TJ. 2007b. Perirhinal cortex resolves feature ambiguity in configural object recognition and perceptual oddity tasks. Learn Mem 14: 821-832.

Beas BS, Setlow B, Bizon JL. 2013. Distinct manifestations of executive dysfunction in aged rats. Neurobiol Aging 34: 2164-2174.
Beas BS, McQuail JA, Banuelos C, Setlow B, Bizon JL. 2016. Prefrontal cortical GABAergic signaling and impaired behavioral flexibility in aged F344 rats. Neuroscience. doi: 10.1016/j.neuroscience.2016.02.014

Bennett IJ, Stark CEL. 2016. Mnemonic discrimination relates to perforant path integrity: an ultra-high resolution diffusion tensor imaging study. Neurobiol Learn Mem 129: 107-112.

Bennett IJ, Huffman DJ, Stark CEL. 2015. Limbic tract integrity contributes to pattern separation performance across the lifespan. Cereb Cortex 25: $2988-2999$.

Bizon JL, LaSarge CL, Montgomery KS, McDermott AN, Setlow B, Griffith WH. 2009. Spatial reference and working memory across the lifespan of male Fischer 344 rats. Neurobiol Aging 30: 646-655.

Burke SN, Barnes CA. 2006. Neural plasticity in the ageing brain. Nat Rev Neurosci 7: 30-40.

Burke SN, Wallace JL, Nematollahi S, Uprety AR, Barnes CA. 2010. Pattern separation deficits may contribute to age-associated recognition impairments. Behav Neurosci 124: 559-573.

Burke SN, Wallace JL, Hartzell AL, Nematollahi S, Plange K, Barnes CA. 2011. Age-associated deficits in pattern separation functions of the perirhinal cortex: a cross-species consensus. Behav Neurosci 125: 836-847.

Burke SN, Hartzell AL, Lister JP, Hoang LT, Barnes CA. 2012. Layer V perirhinal cortical ensemble activity during object exploration: a comparison between young and aged rats. Hippocampus 22: 2080-2093.

Burke SN, Maurer AP, Nematollahi S, Uprety A, Wallace JL, Barnes CA. 2014. Advanced age dissociates dual functions of the perirhinal cortex. J Neurosci 34: 467-480.

Bussey TJ, Saksida LM, Murray EA. 2002. Perirhinal cortex resolves feature ambiguity in complex visual discriminations. Eur J Neurosci 15: $365-374$

Bussey TJ, Saksida LM, Murray EA. 2003. Impairments in visual discrimination after perirhinal cortex lesions: testing "declarative" vs. "perceptual-mnemonic" views of perirhinal cortex function. Eur J Neurosci 17: 649-660.

Campbell KL, Trelle A, Hasher L. 2014. Hyper-binding across time: age differences in the effect of temporal proximity on paired-associate learning. J Exp Psychol Learn Mem Cogn 40: 293-299.

Cansino S. 2009. Episodic memory decay along the adult lifespan: a review of behavioral and neurophysiological evidence. Int J Psychophysiol 71: $64-69$.

de Bruin JP, Sànchez-Santed F, Heinsbroek RP, Donker A, Postmes P. 1994. A behavioural analysis of rats with damage to the medial prefrontal cortex using the Morris water maze: evidence for behavioural flexibility, but not for impaired spatial navigation. Brain Res 652: 323-333.

Devore S, de Almeida L, Linster C. 2014. Distinct roles of bulbar muscarinic and nicotinic receptors in olfactory discrimination learning. J Neurosci 34: $11244-11260$.

DiMattia BD, Kesner RP. 1988. Spatial cognitive maps: differential role of parietal cortex and hippocampal formation. Behav Neurosci 102: 471-480.

Fandakova Y, Lindenberger U, Shing YL. 2014. Deficits in process-specific prefrontal and hippocampal activations contribute to adult age differences in episodic memory interference. Cereb Cortex 24: $1832-1844$.

Gallagher M, Burwell R, Burchinal M. 1993. Severity of spatial learning impairment in aging: development of a learning index for performance in the Morris water maze. Behav Neurosci 107: 618-626.

Gazzaley A, Cooney JW, Rissman J, D’Esposito M. 2005. Top-down suppression deficit underlies working memory impairment in normal aging. Nat Neurosci 8: 1298-1300.

Gilbert PE, Kesner RP. 2006. The role of the dorsal CA3 hippocampal subregion in spatial working memory and pattern separation. Behav Brain Res 169: 142-149.

Gilbert PE, Kesner RP, DeCoteau WE. 1998. Memory for spatial location: role of the hippocampus in mediating spatial pattern separation. J Neurosci 18: 804-810.

Gilbert PE, Kesner RP, Lee I. 2001. Dissociating hippocampal subregions: A double dissociation between dentate gyrus and CA1. Hippocampus 11: $626-636$

Gracian EI, Shelley LE, Morris AM, Gilbert PE. 2013. Age-related changes in place learning for adjacent and separate locations. Neurobiol Aging 34: $2304-2309$.

Gray DT, Barnes CA. 2015. Distinguishing adaptive plasticity from vulnerability in the aging hippocampus. Neuroscience 309: 17-28.

Grön G, Brandenburg I, Wunderlich AP, Riepe MW. 2006. Inhibition of hippocampal function in mild cognitive impairment: targeting the cholinergic hypothesis. Neurobiol Aging 27: 78-87.

Hartman M. 1995. Aging and interference: evidence from indirect memory tests. Psychol Aging 10: 659-669. 
Hasselmo ME. 2006. The role of acetylcholine in learning and memory. Curr Opin Neurobiol 16: 710-715.

Hernandez CM, Gearhart DA, Parikh V, Hohnadel EJ, Davis LW, Middlemore ML, Warsi SP, Waller JL, Terry AV. 2006. Comparison of galantamine and donepezil for effects on nerve growth factor, cholinergic markers, and memory performance in aged rats. JPharmacol Exp Ther 316: 679-694.

Holden HM, Hoebel C, Loftis K, Gilbert PE. 2012. Spatial pattern separation in cognitively normal young and older adults. Hippocampus 22: $1826-1832$.

Holden HM, Toner C, Pirogovsky E, Kirwan CB, Gilbert PE. 2013. Visual object pattern separation varies in older adults. Learn Mem 20: $358-362$.

Jackson S, Ham RJ, Wilkinson D. 2004. The safety and tolerability of donepezil in patients with Alzheimer's disease. Br J Clin Pharmacol 58: $1-8$.

Kesner RP, Gilbert PE. 2006. The role of the medial caudate nucleus, but not the hippocampus, in a matching-to-sample task for a motor response: pattern separation for a motor response. Eur J Neurosci 23: 1888-1894.

Kesner RP, Rolls ET. 2015. A computational theory of hippocampal function, and tests of the theory: new developments. Neurosci Biobehav Rev 48: 92-147.

LaSarge CL, Montgomery KS, Tucker C, Slaton GS, Griffith WH, Setlow B, Bizon JL. 2007. Deficits across multiple cognitive domains in a subset of aged Fischer 344 rats. Neurobiol Aging 28: 928-936.

Lustig C, Hasher L. 2001. Implicit memory is vulnerable to proactive interference. Psychol Sci 12: 408-412.

Marr D. 1971. Simple memory: a theory for archicortex. Philos Trans R Soc Lond B Biol Sci 262: 23-81.

McIntyre JS, Craik FI. 1987. Age differences in memory for item and source information. Can J Psychol 41: 175-192.

McNaughton BL, Morris RGM. 1987. Hippocampal synaptic enhancement and information storage within a distributed memory system. Trends Neurosci 10: 408-415.

McQuail JA, Nicolle MM. 2015. Spatial reference memory in normal aging Fischer $344 \times$ Brown Norway F1 hybrid rats. Neurobiol Aging 36: $323-333$.

Moore TL, Killiany RJ, Herndon JG, Rosene DL, Moss MB. 2003. Impairment in abstraction and set shifting in aged rhesus monkeys. Neurobiol Aging 24: 125-134.

Moore TL, Killiany RJ, Herndon JG, Rosene DL, Moss MB. 2006. Executive system dysfunction occurs as early as middle-age in the rhesus monkey. Neurobiol Aging 27: 1484-1493.

Morris AM, Churchwell JC, Kesner RP, Gilbert PE. 2012. Selective lesions of the dentate gyrus produce disruptions in place learning for adjacent spatial locations. Neurobiol Learn Mem 97: 326-331.

Morrison JH, Baxter MG. 2012. The ageing cortical synapse: hallmarks and implications for cognitive decline. Nat Rev Neurosci 13: 240-250.

Moss MB, Moore TL, Schettler SP, Killiany R, Rosene D. 2007. Successful vs. unsuccessful aging in the rhesus monkey. In Brain aging: models, methods, and mechanisms. Frontiers in neuroscience (ed. Riddle DR). CRC Press, Boca Raton, FL http://www.ncbi.nlm.nih.gov/books/NBK1833/.

O'Reilly RC, Norman KA. 2002. Hippocampal and neocortical contributions to memory: advances in the complementary learning systems framework. Trends Cogn Sci 6: 505-510.

Pettigrew C, Martin RC. 2014. Cognitive declines in healthy aging: evidence from multiple aspects of interference resolution. Psychol Aging 29: $187-204$.

Rajah MN, D'Esposito M. 2005. Region-specific changes in prefrontal function with age: a review of PET and fMRI studies on working and episodic memory. Brain J Neurol 128: 1964-1983.

Reagh ZM, Roberts JM, Ly M, DiProspero N, Murray E, Yassa MA. 2014. Spatial discrimination deficits as a function of mnemonic interference in aged adults with and without memory impairment. Hippocampus 24: $303-314$.

Reagh ZM, Ho HD, Leal SL, Noche JA, Chun A, Murray EA, Yassa MA. 2016. Greater loss of object than spatial mnemonic discrimination in aged adults. Hippocampus 26: 417-422.

Robitsek RJ, Fortin NJ, Koh MT, Gallagher M, Eichenbaum H. 2008. Cognitive aging: a common decline of episodic recollection and spatial memory in rats. J Neurosci 28: 8945-8954.

Rolls ET, Kesner RP. 2006. A computational theory of hippocampal function, and empirical tests of the theory. Prog Neurobiol 79: 1-48.
Rosenzweig ES, Rao G, McNaughton BL, Barnes CA. 1997. Role of temporal summation in age-related long-term potentiation-induction deficits. Hippocampus 7: 549-558.

Rowe G, Hasher L, Turcotte J. 2010. Interference, aging, and visuospatial working memory: the role of similarity. Neuropsychology 24: 804-807.

Ryan L, Cardoza JA, Barense MD, Kawa KH, Wallentin-Flores J, Arnold WT, Alexander GE. 2012. Age-related impairment in a complex object discrimination task that engages perirhinal cortex. Hippocampus 22: 1978-1989.

Santoro A. 2013. Reassessing pattern separation in the dentate gyrus. Front Behav Neurosci 7: 96.

Sapkota RP, van der Linde I, Pardhan S. 2014. How does aging affect the types of error made in a visual short-term memory "object-recall" task? Front Aging Neurosci 6: 346.

Sarter M, Bruno JP. 1997. Trans-synaptic stimulation of cortical acetylcholine and enhancement of attentional functions: a rational approach for the development of cognition enhancers. Behav Brain Res 83: $7-14$.

Schacter DL, Osowiecki D, Kaszniak AW, Kihlstrom JF, Valdiserri M. 1994. Source memory: extending the boundaries of age-related deficits. Psychol Aging 9: 81-89.

Schenk F, Morris RG. 1985. Dissociation between components of spatial memory in rats after recovery from the effects of retrohippocampal lesions. Exp Brain Res 58: 11-28.

Sloan HL, Good M, Dunnett SB. 2006. Double dissociation between hippocampal and prefrontal lesions on an operant delayed matching task and a water maze reference memory task. Behav Brain Res 171: 116-126.

Spangler EL, Waggie KS, Hengemihle J, Roberts D, Hess B, Ingram DK. 1994. Behavioral assessment of aging in male Fischer 344 and brown Norway rat strains and their F1 hybrid. Neurobiol Aging 15: 319-328.

Stark SM, Yassa MA, Stark CEL. 2010. Individual differences in spatial pattern separation performance associated with healthy aging in humans. Learn Mem 17: 284-288.

Stark SM, Yassa MA, Lacy JW, Stark CEL. 2013. A task to assess behavioral pattern separation (BPS) in humans: data from healthy aging and mild cognitive impairment. Neuropsychologia 51: 2442-2449.

Tomás Pereira I, Burwell RD. 2015. Using the spatial learning index to evaluate performance on the water maze. Behav Neurosci 129: 533-539.

Tomás Pereira I, Gallagher M, Rapp PR. 2015. Head west or left, east or right: interactions between memory systems in neurocognitive aging. Neurobiol Aging 36: 3067-3078.

Toner CK, Pirogovsky E, Kirwan CB, Gilbert PE. 2009. Visual object pattern separation deficits in nondemented older adults. Learn Mem 16: $338-342$.

Treves A, Rolls ET. 1992. Computational constraints suggest the need for two distinct input systems to the hippocampal CA3 network. Hippocampus 2: 189-199.

Vafaei AA, Rashidy-Pour A. 2004. Reversible lesion of the rat's orbitofrontal cortex interferes with hippocampus-dependent spatial memory. Behav Brain Res 149: 61-68.

van der Staay FJ, Blokland A. 1996. Behavioral differences between outbred Wistar, inbred Fischer 344, brown Norway, and hybrid Fischer $344 \times$ brown Norway rats. Physiol Behav 60: 97-109.

Wais PE, Gazzaley A. 2014. Distractibility during retrieval of long-term memory: domain-general interference, neural networks and increased susceptibility in normal aging. Front Psychol 5: 280.

Wiig KA, Burwell RD. 1998. Memory impairment on a delayed non-matching-to-position task after lesions of the perirhinal cortex in the rat. Behav Neurosci 112: 827-838.

Xavier GF, Oliveira-Filho FJ, Santos AM. 1999. Dentate gyrus-selective colchicine lesion and disruption of performance in spatial tasks: difficulties in "place strategy" because of a lack of flexibility in the use of environmental cues? Hippocampus 9: 668-681.

Yassa MA, Lacy JW, Stark SM, Albert MS, Gallagher M, Stark CEL. 2011a. Pattern separation deficits associated with increased hippocampal CA3 and dentate gyrus activity in nondemented older adults. Hippocampus 21: $968-979$.

Yassa MA, Mattfeld AT, Stark SM, Stark CEL. 2011b. Age-related memory deficits linked to circuit-specific disruptions in the hippocampus. Proc Natl Acad Sci 108: 8873-8878.

Received March 1, 2016; accepted in revised form April 10, 2016. 


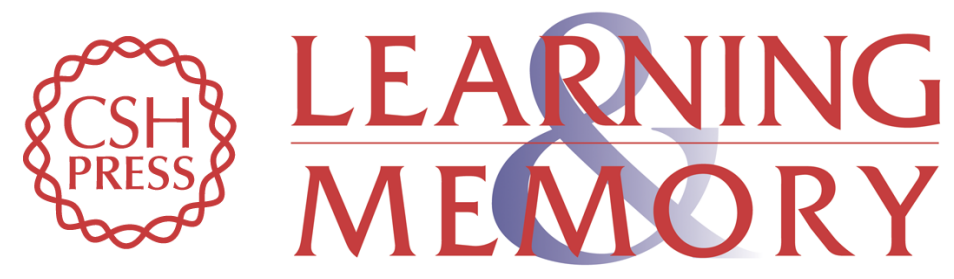

\section{Discrimination performance in aging is vulnerable to interference and dissociable from spatial memory}

Sarah A. Johnson, Patricia K. Sacks, Sean M. Turner, et al.

Learn. Mem. 2016, 23:

Access the most recent version at doi:10.1101/Im.042069.116

\section{Supplemental http://learnmem.cshlp.org/content/suppl/2016/06/15/23.7.339.DC1 Material}

References This article cites 80 articles, 13 of which can be accessed free at: http://learnmem.cshlp.org/content/23/7/339.full.html\#ref-list-1

Creative This article is distributed exclusively by Cold Spring Harbor Laboratory Press for the Commons first 12 months after the full-issue publication date (see

License http://learnmem.cshlp.org/site/misc/terms.xhtml). After 12 months, it is available under a Creative Commons License (Attribution-NonCommercial 4.0 International), as described at http://creativecommons.org/licenses/by-nc/4.0/.

Email Alerting Receive free email alerts when new articles cite this article - sign up in the box at the Service top right corner of the article or click here. 